\title{
CENTRAL ADMINISTRATION OF GALANIN N- TERMINAL FRAGMENT 1-15 DECREASES THE VOLUNTARY ALCOHOL INTAKE IN RATS.
}

Carmelo Millón ${ }^{1 *}$, Antonio Flores-Burgess ${ }^{1}$, Estela Castilla-Ortega ${ }^{2}$, Belén Gago1, María García-Fernandez¹, Antonia Serrano², Fernando Rodriguez de Fonseca², José Angel Narváez¹, Kjell Fuxe³ Luis Santín ${ }^{4}$ \& Zaida Díaz-Cabiale ${ }^{1}$.

1.Universidad de Málaga, Instituto de Investigación Biomédica de Málaga, Facultad de Medicina, Campus de Teatinos s/n, 29071 Málaga, Spain.

2. Unidad de Gestión Clínica de Salud Mental e Instituto de Investigación Biomédica de Málaga, Hospital Regional Universitario de Málaga, Málaga 29010, Spain

3. Department of Neuroscience, Karolinska Institute, Stockholm, Sweden. 4.Universidad de Málaga, Instituto de Investigación Biomédica de Málaga, Facultad de Psicología, Campus de Teatinos s/n, 29071 Málaga, Spain

"Corresponding author:

Carmelo Millón Peñuela, PhD

Departamento de Fisiología

Facultad de Medicina

Universidad de Málaga

Campus de Teatinos s/n. 29080 Málaga, Spain

E-mail: carmelomp@uma.es 


\section{ABSTRACT}

Alcohol consumption is considered a major risk factor for disease and mortality worldwide. In the absence of effective treatments in alcohol use disorders (AUD) it is important to find new biological targets that could modulate alcohol consumption. We tested the role of the N-terminal Galanin fragment (1-15) [GAL(1-15)] in voluntary ethanol consumption in rats using the two-bottle choice paradigm as well as compare the effects of GAL(1-15) with the whole molecule of Galanin (GAL). We describe for the first time that GAL(1-15), via central mechanisms, induces a strong reduction in preference and ethanol consumption in rats. These effects were significantly different than GAL. Galanin receptor (GALR) 2 was involved in these effects, since the specific GALR2 antagonist M871 blocked GAL(1-15) mediated actions in preference and ethanol intake. Importantly, the mechanism of this action involves changes in GALR expression and also in immediate-early gene C-Fos and receptorsinternalization-related gene Rab5 in the striatum. The relevance of the striatum as a target for $\operatorname{GAL}(1-15)$ was supported by the effect of $G A L(1-15)$ on the locomotor activity of rats after ethanol administration. These results may give the basis for the development of novel therapeutics strategies using GAL(1-15) analogues for the treatment of AUD in humans.

Key Words: Galanin, Galanin (1-15), Alcohol, Addiction 


\section{INTRODUCTION}

Alcohol consumption is considered a major risk factor for disease and mortality worldwide according to the World Health Organization (2014). Current therapies in alcohol use disorders (AUD) have limited efficacy, produce several adverse effects and present high rates of relapse. In the absence of effective treatments it is therefore of great importance to find new biological targets that could modulate alcohol consumption. Numerous neurotransmitters have been implicated in AUD including GABA, glutamate, dopamine, noradrenaline, serotonin and several endogenous neuropeptides (Schneider et al., 2007; Marcinkiewcz et al., 2016).

Galanin (GAL) is a neuropeptide (Tatemoto et al., 1983) widely distributed in neurons within the central nervous system (CNS) (Jacobowitz et al., 2004). Three GAL receptor (GALR1-3) subtypes with high affinities for GAL have been cloned (Branchek et al., 2000; Mitsukawa et al., 2008). GALR1 and GALR3 mainly activate inhibitory $\mathrm{G}$ proteins $\mathrm{Gi} / \mathrm{Go}$, while GALR2 mainly couples to $\mathrm{Gq} / \mathrm{G} 11$ to mediate excitatory signalling (Branchek et al., 2000).

GAL participates in a number of central functions modulating neuroendocrine levels, pain control, cardiovascular functions, food intake and mood disorders (Mitsukawa et al., 2008; Diaz-Cabiale et al., 2010; Lang et al., 2015). GAL and their receptors are also involved in drug abuse and addiction (Picciotto, 2008), including alcohol intake and alcoholism (Lewis et al., 2004; Lewis et al., 2005). Microinjection of GAL into the third ventricle increased ethanol consumption in Sprage-Dawley rats in two-bottle choice test from a 7\% ethanol solution over water, and this increase was completely reversed with the GAL receptor antagonist M40 (Lewis et al., 2004). These effects of GAL over ethanol consumption were also found with microinjections of GAL directly into the paraventricular nucleus (PVN) of the hypothalamus (Rada et al., 2004). Moreover, mice overexpressing GAL show an increase in preference and ethanol intake in comparison with their wild-type peers (Karatayev et al., 2009), while GAL knockout mice drink less ethanol and decrease the preference for it (Karatayev et al., 2010). Not only the PVN but also the reward circuitry seems to be involved in the effects of GAL promoting alcohol drinking. Injection of GAL 
in the PVN can increase dopamine release in the nucleus accumbens (NAc) (Rada et al., 1998), and this effect would be consistent with the ability of GAL to increase the rewarding effects of alcohol (Picciotto et al., 2010).

In addition to GAL, also the N-terminal fragments like $\mathrm{GAL}(1-15)$ are active in the CNS (Hedlund et al., 1996; Diaz-Cabiale et al., 2005; Diaz-Cabiale et al., 2010; Millon et al., 2015; Millon et al., 2016; Flores-Burgess et al., 2017; Millon et al., 2017). Both GAL and GAL(1-15) molecules have specific roles in cardiovascular regulation and interact differently with other neuropeptides (DiazCabiale et al., 2005). Recently we have described that GAL(1-15) induces a strong depression-related and anxiogenic-like effects in rats and these effects were significantly stronger than the ones induced by GAL. The GALR1/GALR2 heteroreceptor complexes in the dorsal hippocampus and especially in the dorsal raphe, areas rich in GAL(1-15) binding sites (Hedlund et al., 1992), were involved in these effects (Millon et al., 2015). The presence of specific binding sites for GAL(1-15) in the dorsal hippocampus, neocortex and striatum (Hedlund et al., 1992), which is part of mesolimbic dopamine system (Koob, 1992), suggest a role of GAL(1-15) in the circuits related to the rewarding and motivational effects of drugs of abuse.

The purpose of the current study was to assess the role of intracerebroventricular (i.c.v.) GAL(1-15) in voluntary ethanol consumption in rats using the two-bottle choice paradigm as well as compare the effects of GAL(1-15) with the GAL. Moreover, the involvement of GALR2 in GAL(1-15)mediated effects in this test was analysed with the selective GALR2 antagonist M871. In order to investigate whether the effect of GAL(1-15) in voluntary ethanol consumption was associated with the reward-circuit, we have studied in the striatum the effect of GAL(1-15) in the expression of the immediate-early gene C-Fos, the receptors-internalization related gene Rab5 and the GALR1 and GALR2. We therefore investigated the effects of $G A L(1-15)$ treatment on the locomotor activity induced by alcohol. 


\section{MATERIAL AND METHODS}

\section{Animals}

Male Sprague Dawley rats (body weight 225-250g, age 8 weeks) were obtained from Criffa and maintained in a humidity- and temperature-controlled $\left(20-22^{\circ} \mathrm{C}\right)$ room. The rats in the two-bottle choice paradigm were during the entire protocol maintained on a 12-hour reversed light/dark cycle (lights off at 10 am) whereas the other rats kept on 12-/hour light/dark cycle. The animals had free access to food pellets and tap water. All animal experimentation was conducted in accordance with the University of Málaga Guidelines for the Care and Use of Laboratory Animals.

\section{Intracerebroventricular Injections}

This protocol has been used previously (Diaz-Cabiale et al., 2011; Millon et al., 2015). Briefly, the rats were anesthetized intraperitoneally with Equitesin (21.3g choral hydrate, $10.6 \mathrm{~g}$ magnesium sulphate and $4.8 \mathrm{~g}$ phenobarbitone dissolved in $500 \mathrm{~mL}$ destilated water containing $9.5 \%$ ethanol and propylene glycol ;3.3mL/kg body weight), and stereotaxically implanted with a unilateral chronic 22-gauge stainless-steel guide cannula into the right lateral cerebral ventricle using the following coordinates: $1.4 \mathrm{~mm}$ lateral and $1 \mathrm{~mm}$ posterior to bregma, and $3.6 \mathrm{~mm}$ below the surface of the skull (Paxinos, 1986). After surgery, animals were individually housed and allowed a recovery period of 7 days. The injections in the lateral ventricle were performed using a 26-gauge stain less-steel injection cannula connected via a PE-10 tubing to a Hamilton syringe. The total volume was $5 \mu$ per injection and the infusion time was $1 \mathrm{~min}$.

Solutions were prepared freshly and the peptides were dissolved in artificial cerebrospinal fluid (composition is $120 \mathrm{nM} \mathrm{NaCl}, 20 \mathrm{nM} \mathrm{NaH} 2 \mathrm{CO} 3,2 \mathrm{nM} \mathrm{KCl}$, 0.5nM KH2PO4, 1.2nM CaCl2, 1.8 nM MgCl2, 0.5 nM Na2SO4, and 5.8 nM Dglucose, $\mathrm{pH}$ 7.4). GAL was obtained from NeoMPS, Strasbourg, France; GAL(1-15) and the GALR2 receptor antagonist M871 were obtained from Tocris Bioscience,Bristol,UK. 


\section{Two-bottle choice test for voluntary ethanol consumption and preference}

The two-bottle choice test was used to determine the voluntary ethanol consumption of rats as described previously (Castilla-Ortega et al., 2016). Briefly, after 7 days of water consumption (both bottles), a choice between water and increasing ethanol concentration $[3,6$ and $10 \%(\mathrm{v} / \mathrm{v})]$ was offered for 7 days each. A choice between $10 \%(\mathrm{v} / \mathrm{v})$ ethanol and water was offered for several days until reaching a stable baseline. Water and ethanol consumption were recorded daily. The bottles positions were changed every day to avoid position preferences. Water intake $(\mathrm{g} / \mathrm{Kg})$, ethanol intake $(\mathrm{g} / \mathrm{Kg})$ and preference [ethanol consumption/total fluid (water plus ethanol) consumption $\times 100$ ] were calculated for each animal. Throughout the experiment, evaporation/spillage estimates were calculated using an empty cage with two bottles, one containing water and the other containing the appropriate ethanol solution. In the experiments a choice between ethanol (10\%) and water was offered.

Three sets of experiments were conducted in the two-bottle choice paradigm. In the first set of experiments, a dose-response curve of GAL(1-15) was performed. For this, groups of rats received i.c.v. GAL(1-15) $1 \mathrm{nmol}, 3 \mathrm{nmol}$ or vehicle 2, 14 and 24 hours before the measures. In the second set of experiments, the effects in two-bottle choice test of GAL and GAL(1-15) were compared. For this, groups of rats received i.c.v. GAL $3 \mathrm{nmol}, \mathrm{GAL}(1-15) 3 \mathrm{nmol}$ or vehicle 2, 14 and 24 hours before the test. In the last set of experiments, the role of the GALR2 was studied; for this groups of rats received i.c.v. GAL(1-15) 3nmol combined with GALR2 antagonist M871 $3 \mathrm{nmol} 2$ hours before the measures.

The general schema of the experimental design is shown in Fig.1.

mRNA Expression of Galanin receptors, C-Fos and Rab5 genes in the striatum in voluntary ethanol consumption

Groups of rats from the two-bottle choice paradigm were killed by decapitation 2 hours after a single i.c.v. administration of GAL(1-15) $3 \mathrm{nmol}$ or vehicle. The rats had free access to ethanol and water until the moment of sacrifice. The corpus striatum was manually dissected, including both the dorsal and ventral areas 
from $2.2 \mathrm{~mm}$ anterior to bregma to $1.8 \mathrm{~mm}$ to bregma (Paxinos, 1986) and frozen on solid $\mathrm{CO}_{2}$ until mRNA expression analysis.

RNA isolation and quantitative real-time $P C R$ analysis

The procedure to perform RNA isolation and RT-PCR was described previously (Millon et al., 2015). Total RNA was isolated from the striatum using RNeasy Lipid Tissue Kit (Qiagen, Hilden, Germany). cDNA was obtained using a Reverse Transcriptase Core Kit (Eurogentec, Seraing, Belgium). These steps were performed according to the manufacture's instructions.

All PCR were conducted in triplicate using Power SYBR Green PCR Master Mix (Applied Biosystems, Foster City, USA) in 7500 RT-PCR system (Applied Biosystems, Foster City, USA). The primers sequences used in this study are:

GAPDH-Forward: 5'-GCTCTCTGCTCCTCCCTGTTC; GAPDH-Reverse:5'GAGGCTGGCACTGCACAA;

GALR1-Forward: 5'-AAAACTGGACAAAACTTAGCC; GALR1-Reverse: 5'GGATACCTTTGTCTTTGCTC;

GALR2-Forward: 5'-AACAGGAATCCACAGACC; GALR2-Reverse:5'CCCTTTGGTCCTTTAACAAG ;

C-FOS-Fordward: 5'-AAACGGAGAATCCGAAGG; C-FOS-Reverse:5'CGTCTTCAAGTTGATCTGTC;

RAB5-Fordward: 5'-AAAAGAGCTGTTGACTTCC; RAB5-Reverse: 5'AGGTCTACTCCTCTTCCTC. The data were analyzed using the comparative $\mathrm{Ct}$ method and normalized to measures of glyceraldehyde-3-phosphate dehydrogenase (GAPDH) mRNA

\section{Alcohol dehydrogenase assay}

The activity of hepatic cytosolic alcohol dehydrogenase (ADH) in the rats from the two-bottle choice test was analysed. ADH activity has a good correlation with ethanol elimination rates in vivo (Lumeng et al., 1979) and chronic ethanol administration causes an increase in ADH activity (Buris et al., 1985).

The livers were removed 2 hours after i.c.v. injections and frozen in solid $\mathrm{CO}_{2}$ until use. Determination of $A D H$ activity was perform in homegenated of liver tissue in Tris $\mathrm{HCl}$ solution $(10 \mathrm{mM} \mathrm{pH} 8,80.5 \mathrm{mM}$ dithiothreitol) centrifuged at 
$12.000 \mathrm{~g}$ during $10 \mathrm{~min}$. ADH activity was evaluated using a microassay adapted to Cobas Mira according to Shephard and colleges (Shephard et al., 1987). Briefly, $300 \mu \mathrm{L}$ of NAD solution ( $2.9 \mathrm{mM}$ in $0.1 \mathrm{M}$ Glycine/ $\mathrm{NaOH} \mathrm{pH} 10$ ) was added to $30 \mu \mathrm{L}$ of sample. The first optical reading was recorded before the addition of $20 \mu \mathrm{L}$ of $17 \mathrm{mM}$ ethanol and then the rate of change in absorption of the NADH chromogen is monitored at $340 \mathrm{~nm}$ over time on the Cobas Mira at $37{ }^{\circ} \mathrm{C}$. One activity unit was defined as the reduction of $1 \mathrm{~mol}$ NAD to $\mathrm{NADH} / \mathrm{min}$ at $37^{\circ} \mathrm{C}$.

\section{Locomotor activity experiments}

In this experiment the effects of $\mathrm{GAL}(1-15)$ (3nmol) on ethanol-reduced locomotion (1.75 g/Kg; intraperitoneal (i.p.)) (Vallof et al., 2016) were investigated. Locomotor activity was registered in the rat open field $(100 \times 100 \times$ $50 \mathrm{~cm}$ ) where animals were individually placed and allowed to freely explore. Their behaviour was recorded over $30 \mathrm{~min}$ period by ceiling-mounted video camara and locomotor activity was analyzed using the video-tracking software EthovisionXT. After each trial, all surfaces were cleaned with a paper towel and $70 \%$ ethanol solution. For the locomotor activity, the total distance travelled $(\mathrm{cm})$ and mean speed $(\mathrm{cm} / \mathrm{s})$ were recorded. Groups of rats were administrated via i.c.v. with $\mathrm{GAL}(1-15)$ or vehicle $20 \mathrm{~min}$ before the test and the i.p. ethanol $(1.75 \mathrm{~g} / \mathrm{Kg})$ or saline administration was 5 min prior the test.

\section{Expression of mRNA of GALR1 and GALR2 after acute administration of ethanol}

Groups of naïve rats were injected i.p. with ethanol $4 \mathrm{~g} / \mathrm{Kg}(50 \% \mathrm{v} / \mathrm{v} 10 \mathrm{~mL} / \mathrm{Kg})$ (Bilbao et al., 2016), dissolved in sterile saline $0.9 \%(\mathrm{w} / \mathrm{v})$, and 0,2 and 4 hours after injection the brains were collected after a rapid decapitation. The corpus striatum was manually dissected, including both the dorsal and ventral areas from $2.2 \mathrm{~mm}$ anterior to bregma to $1.8 \mathrm{~mm}$ posterior to bregma (Paxinos, 1986) and frozen immediately on solid $\mathrm{CO}_{2}$ until use. The procedure to perform RNA isolation and RT-PCR was described previously. 


\section{Statistical Analysis}

Data are presented as the means \pm standard error of the mean and sample numbers $(n)$ are indicated in figure legends. All data were analyzed using GraphPad PRISM 4.0 (GraphPad Software). For comparing two experimental conditions, student's unpaired t-test statistical analyses were performed. Otherwise, one-way analyses of variance (ANOVAs) or two-way ANOVAs followed by Fisher's LSD comparison post-tests were performed. Differences were considered significant at $p<0.05\left({ }^{*} p<0.05,{ }^{* *} p<0.01,{ }^{* * *} p<0.001\right)$.

\section{RESULTS}

\section{Liver ADH activity in voluntary ethanol consumption}

Voluntary ethanol intake through two-bottle choice test protocol induced a significant increased in the ADH activity compared with baseline group $\left(t_{11}=3.281 p<0.01\right)$, confirming the validity of our model (Table 1$)$.

GAL(1-15) induced a decrease of ethanol intake and alcohol preference in the two-bottle choice paradigm

GAL(1-15) at $3 \mathrm{nmol}$ significantly decreased the ethanol intake at 2 hours (oneway ANOVA, F2,30=3.54 $p<0.05$, Fisher's LSD post-hoc test: $p<0.05$; Fig. $2 a$ ), 14 hours (one-way ANOVA, F2,30=3.44 $p<0.05$, Fisher's LSD post-hoc test: $p<0.05$; Fig. $2 b$ ) and 24 hours (one-way ANOVA, F2,29=3.59 $p<0.05$, Fisher's LSD post-hoc test: $p<0.05$; Fig. $2 c$ ) after its administration. GAL(1-15) at the dose of $1 \mathrm{nmol}$ lacked effect on ethanol intake in all the time-points analysed.

Moreover, 2 hours after i.c.v. GAL(1-15) $3 \mathrm{nmol}$ a significantly decreased by $90 \%$ in preference was observed (one-way ANOVA, F2,31=3.46 $p<0.05$, Fisher's LSD post-hoc test: $p<0.05$; Fig. $2 \mathrm{~g}$ ). This effect was maintained 24 hours (one-way ANOVA, F2,31=3.57 $p<0.05$, Fisher's LSD post-hoc test: $\mathrm{p}<0.05$; Fig. 2i). The dose of $\operatorname{GAL}(1-15)$ 1nmol lacks again of effects in preference. 
In the water (Fig. 2d,e,f) and food intake (Fig. 2j,k,I), no differences were found at any time-point after GAL(1-15) i.c.v. administration at any dose.

These results indicate that GAL(1-15) evokes a strong decrease in voluntary ethanol intake and preference in rats.

\section{Comparison between GAL and GAL(1-15) in the two-bottle choice paradigm}

In the ethanol intake, the overall one-way ANOVA revealed a significant difference between the N-terminal fragment GAL(1-15) and GAL at 2, 14 and 24 hours after treatments. Two hours after the injection, $\operatorname{GAL}(1-15)$ significantly reduced the ethanol intake compared to GAL (one-way ANOVA, F2,31=4.208 $p<0.05$, Fisher's LSD post-hoc test: $p<0.01$; Fig. 3a). The same pattern of response was observed at the others times, GAL(1-15) significantly decreased the ethanol intake versus GAL groups 14 (one-way ANOVA, F2,30=3.97 $p<0.05$, Fisher's LSD post-hoc test: $p<0.05$; Fig. $3 b$ ) and 24 hours (one-way ANOVA, $F 2,30=2.53 p<0.05$, Fisher's LSD post-hoc test: $p<0.05$; Fig. $3 c$ ) after i.c.v. injection.

On the preference, the difference between GAL and GAL(1-15) was observed again. GAL(1-15) decreased the preference compared with GAL 2 hours after its administration (one-way ANOVA, F2,30=3.55 p<0.05, Fisher's LSD post-hoc test: $p<0.05$; Fig. $3 g$ ), effect that was maintained 24 hours later (one-way ANOVA, F2,32=3.43 $p<0.05$, Fisher's LSD post-hoc test: $p<0.05$; Fig. 3i).

GAL lacks effect on the ethanol intake and preference compared to the vehicle at any time point (Fig. 3).

No differences were found between GAL and the fragment GAL(1-15) in water intake (Fig. 3d, e, f) and food intake (Fig. 3j, k, I).

GALR2 Antagonist M871 Blocked GAL(1-15)-mediated effects in the twobottle choice paradigm 
In the two-bottle choice test, GALR2 antagonist M871 significantly blocked the decreased in the ethanol intake (one-way ANOVA, F2,34=3.72 $p<0.05$, Fisher's LSD post-hoc test: $p<0.05$; Fig. $4 a$ ) induced by $\operatorname{GAL}(1-15) 2$ hours after its administration.

In the preference the same type of change was observed. Thus, GALR2 participates in GAL(1-15)-mediated effect, since the GALR2 antagonist M871 significantly blocked the decreased on preference induced by GAL(1-15) (oneway ANOVA, F2,32=3.39 p<0.05, Fisher's LSD post-hoc test: $p<0.05$; Fig. $4 c$ ).

The GALR2 antagonist M871 alone at the dose of $3 \mathrm{nmol}$ lacked effects on ethanol intake $(0.13 \pm 0.06 \mathrm{~g} / \mathrm{Kg})$ and preference $(12.65 \pm 8.23 \%)$.

Neither water (Fig. 4b) nor food intake (Fig. 4d) were modified by M871 (water intake: $8.26 \pm 2.00 \mathrm{~g} / \mathrm{Kg}$; food intake: $6.67 \pm 0.95 \mathrm{~g}$ ) or GAL(1-15)+M871 2 hours after injection.

GAL(1-15) effects mRNA Expression of Galanin receptors, C-Fos and Rab5 genes in the striatum in voluntary ethanol consumption

As shown in Fig. 5, GAL(1-15) at dose of $3 \mathrm{nmol}$ produced a significant increase in the mRNA levels of C-Fos ( $\mathrm{t}_{8}=5.488 \mathrm{p}<0.001$;Fig. $5 \mathrm{a}$ ) and Rab5 $\left(\mathrm{t}_{6}=4.148\right.$ $p<0.01$;Fig. $5 b$ ) in the two-bottle choice test 2 hour after its administration.

The administration of GAL(1-15) also modified the GALR1 and GALR2 receptors expression in striatum, producing a significant decreased of GALR1 mRNA levels ( $\mathrm{t}_{10}=2.341 \mathrm{p}<0.05$; Fig. $5 \mathrm{c}$ ) and a slight reduction in GALR2 expression ( $\mathrm{t}_{10}=1.360 \mathrm{p}=0.101$; Fig. $5 \mathrm{~d}$ ), suggesting the involvement of both receptors in the effects of GAL(1-15).

\section{Effects of GAL(1-15) on ethanol-reduced locomotion}

As previously described, the i.p. administration of ethanol $1.75 \mathrm{~g} / \mathrm{kg}$ reduced the distance travelled (one-way ANOVA, F1,25=62.2 $p<0.001$ ) and the mean speed 
(one-way ANOVA, $\mathrm{F} 1,25=62.2 \mathrm{p}<0.001$ ) five minutes after its administration (Fig. 6).

The effect of GAL(1-15) $3 \mathrm{nmol}$ on locomotion (distance travelled and mean speed), was dependent on i.p. ethanol (distance travelled: two-way ANOVA for alcohol/i.c.v.-treatment interaction $\mathrm{F} 1,25=7.19 \mathrm{p}<0.01$; mean speed two-way ANOVA for alcohol/i.c.v.-treatment interaction $\mathrm{F} 1,25=7.19$ p<0.01) (Fig. 6). Thus, in rats following i.p. administration of ethanol, GAL(1-15) decreased the distance travelled (Fisher's LSD post-hoc test: $p<0.001$; Fig. $6 a$ ), whereas no such effects were observed in rats with systemic administration of saline (Fisher's LSD post-hoc test: $\mathrm{p}=0.71$; Fig. $6 \mathrm{a}$ ). Similar to the distance travelled, GAL(1-15) decreased the mean speed only in the rats systemically treated with ethanol (Fisher's LSD post-hoc test: $p<0.001$; Fig. $6 b$ ).

\section{GALR1 and GALR2 expression in the striatum after the acute administration of ethanol}

To determine whether ethanol influenced GALR1 and GALR2 expression in the striatum, we evaluated the effects of the acute ethanol administration on mRNA GALR1 and GALR2 levels in the striatum at 2 and 4 hours.

As shown in Figure 7, a single ethanol injection ( $4 \mathrm{~g} / \mathrm{Kg}$ i.p.) lacked effect in the GALR1 expression in the striatum 2 and 4 hours after administration (one-way ANOVA, $F 2,14=1.19 p=0.24$ )

No effects were observed also in the GALR2 mRNA levels in any of the timepoints (one-way ANOVA, F2,14=1.15 p=0.34).

\section{DISCUSSION}

In the current study, we described for the first time that GAL(1-15), via central mechanisms, induces a strong reduction in preference and ethanol consumption in rats. These effects were significantly different than GAL, showing a differential role of GAL compared with $G A L(1-15)$ in alcohol consumption behaviour. GALR2 was involved in these effects, since the specific 
GALR2 antagonist M871 blocked GAL(1-15) mediated actions in preference and ethanol intake. Importantly, the mechanism of this action involves changes in GAL receptors expression and also in immediate-early gene C-Fos and receptors-internalization-related gene Rab5 in striatum, area rich in GALfragment binding sites (Hedlund et al., 1992) and critical for the rewarding and motivational effects of drugs of abuse (Koob, 1992). The relevance of the striatum as a target for GAL(1-15) was also supported by the effect of GAL(1$15)$ on the locomotor activity of rats after ethanol administration.

GAL(1-15) at the dose of $3 \mathrm{nmol}$ induced a strong reduction in preference and ethanol consumption in the two-bottle choice test at 2 hours, effect that was maintained at 24 hours. Given that this alcohol two-bottle-choice drinking paradigm induces voluntary intake of high amounts of alcohol (Simms et al., 2008), the present data may suggest that GAL(1-15) could be used as a pharmacological agent to treat AUD in humans.

Although emotional states as anxiety are relevant variables to modulate alcohol-taking behaviour, increasing alcohol consumption and preference (Chappell et al., 2013), and GAL(1-15) increases anxiety- and depressive-like behaviours in rats (Millon et al., 2015; Millon et al., 2017), the fact that GAL(115) induced a reduction in alcohol intake and preference suggest that the mechanism involved is independent of the effect of GAL(1-15) in the emotional states.

In addition, a tentative explanation for the reduced alcohol intake induced by central $\operatorname{GAL}(1-15)$ administration, is that $\operatorname{GAL}(1-15)$ by itself may induce aversion rather than attenuate the rewarding properties of alcohol. However, the selected doses of GAL(1-15) lack effect on water and food intake suggesting that the reduced alcohol intake is not driven by aversion to alcohol.

Since GAL(1-15) did not modify the food intake in rats that had been chronically consuming ethanol, it can also be suggested that in our model, the rats were not consuming ethanol just for its calories (Lewis et al., 2004).

Furthermore, the effects of $\operatorname{GAL}(1-15)$ in the two-bottle choice test were significantly different than the corresponding effects induced by GAL. In our 
model GAL $3 \mathrm{nmol}$ lacked effect with respect to vehicle group in all the parameters studied. In previous studies, GAL microinjected into the third ventricle increased the $7 \%$ ethanol intake in the two-bottle choice test (Lewis et al., 2004), and this increase was stronger during the light phase, when the animals are inactive and normally drink very little (Lewis et al., 2004). The different results found in the present work could be explained in the differences of the percentage of ethanol and the light-dark cycle used, since we used the choice between $10 \%$ ethanol versus water, and the percentage of ethanol solution could affect the result of the test (Leeman et al., 2010; Tarragon et al., 2012). Moreover, we performed the measures in the dark period, when the rats are more active, while the ethanol intake produced by GAL was stronger in the light period (Lewis et al., 2004).

However, since GAL(1-15) reduced the preference and ethanol consumption, opposite effect found by GAL by other authors, our results validate and extend the view of a specific role of GAL(1-15) in ethanol intake.

We have previously described a different action of GAL and GAL(1-15) in behaviour functions (Millon et al., 2017). GAL(1-15) induces depression-related and anxiogenic-like effects in rats, and these effects were significantly stronger than the one induced by GAL (Millon et al., 2015). GAL(1-15) is also able to enhance the antidepressant effects induced by the 5HT1A agonist 8-OH-DPAT in the Forced Swimming Test, an effect that was again significantly stronger than the one induced by GAL (Millon et al., 2016). The different action between GAL and GAL(1-15) was observed not only in behavioural functions, but also in central cardiovascular regulation (Diaz-Cabiale et al., 2005; Diaz-Cabiale et al., 2010). Our results in the ethanol intake confirm a unique action of $\operatorname{GAL}(1-15)$ in brain communication.

The mechanism that explained the differences between the GAL, and GAL(1$15)$ is that this N-terminal GAL fragment preferring site is the result of formation of GALR1/GALR2 heteromers highly specific for GAL fragments (Fuxe et al., 2008; Fuxe et al., 2012; Millon et al., 2015). The fact that GALR2 antagonist M871 blocked the reduction in preference and ethanol intake induced by 
GAL(1-15), confirms that GAL(1-15) acts through heterodimer GALR1/GALR2 to reduce the preference and ethanol consumption.

The GAL receptors involved in alcoholism are not well characterized; however, several studies suggested that GALR3 was involved in alcohol consumption (Belfer et al., 2007; Ash et al., 2011; Ash et al., 2014; Scheller et al., 2017). Since GALR3 is mainly restricted to the hypothalamus and pituitary (Smith et al., 1998; Waters et al., 2000) it has been proposed that these areas are crucial for this effect. Our results demonstrate the importance of GALR1 and GALR2 in the reduction of preference and ethanol intake induced by $\mathrm{GAL}(1-15)$ and suggest that the striatum, a key region in the reward effects of drugs (Koob, 1992), is involved in GAL(1-15)-mediated effects. In our voluntary ethanol consumption model, GAL(1-15) induced a significant increase of C-Fos mRNA and Rab5 expression in the striatum suggesting an enhancement of neuronal activation and receptor internalization in this area (Borroto-Escuela et al., 2012). Moreover, in these animals, we observed after $\mathrm{GAL}(1-15)$ injection a significant reduction of GALR1 expression and a slight decrease in GALR2 mRNA in the striatum suggesting that both striatal receptors participated in the GAL(1-15)-mediated effects on the voluntary ethanol intake in this nucleus. Interestingly, the evidence that acute ethanol injections in naïve animals lacks of effect in GALR1 and GALR2 expression in the striatum, indicates that ethanol per se do not influence GALR expression and confirm that the effects at GALR level in our model were induced by $\mathrm{GAL}(1-15)$.

The relevance of the striatum as a target for $\mathrm{GAL}(1-15)$ was supported by the the ability of GAL(1-15) to enhance the suppression of locomotor activity induced by ethanol. Ethanol suppression of locomotor activity following ethanol i.p. injection is a well-known behavioural effect mainly mediated by the dopaminergic system, involving the striatum. Accordingly, it has been demonstrated that this effect is notably reduced when the dopaminergic system is lesioned using 6-OHDA, producing strong dopamine depletion in the striatum (Breese et al., 1984). Our results suggest, that the potentiation of the hypolocomotion induced by GAL(1-15) in rats treated with alcohol, may require the modulation of the dopaminergic system by GAL(1-15), probably involving the striatum because it is a crucial target of the dopaminergic projections. 
Although previously, the increase in the ethanol intake of GAL was related directly with several areas within the hypothalamus (Leibowitz et al., 2003; Rada et al., 2004; Schneider et al., 2007), the current work suggests that GAL(1-15) act through the striatum, an area which has specifics binding sites for GAL(1-15) (Hedlund et al., 1992). The involvement of the striatum in GAL(115)-mediated action would explain the different action between GAL and GAL(1-15) in ethanol intake.

Further studies are needed to describe the neurochemical pathways involved in the reduction of ethanol consumption by GAL(1-15); however the dopamine should be consider a target neurotransmitter in this effect. Supporting this hypothesis, GAL reduces behavioural response following treatment with several addictive substances such as morphine or amphetamines mainly modulating dopaminergic neurotransmission (Tsuda et al., 1998; Pierce et al., 2006) and dopamine transmission plays a crucial role in the motor effects of alcohol in the striatum (Brabant et al., 2014).

In conclusion, our results indicate that the N-terminal fragment GAL(1-15) induces a strong reduction in preference and ethanol consumption in rats probably with the involvement of the striatum, a key region in the reward effects of drugs. These results may give the basis for the development of novel therapeutics strategies using GAL(1-15) analogues for the treatment of AUD in humans.

\section{Acknowledgements}

This study was supported by grants awarded by Spanish Ministry of Economy (SAF2016-79008-P), and by University of Málaga (Proyecto Jovénes Investigadores PPIT.UMA.B1.2017/17). C.M was supported by a fellowship from University of Málaga (Contrato Postoctoral UMA) and Junta de Andalucía (Contrato Posdoctoral de Excelencia Proyecto CVI-6476).

\section{Authors Contribution}


$\mathrm{CM}$ was responsible for the study concept and design, conducted the experiments, analysed the data and drafted the manuscript. AFB and BG contributed to the acquisition of animal data. MGF performed the ADH analysis. ECO, AS, FRF, JAN and KF provided critical revision of the manuscript for important intellectual content. LS assisted with data analysis and interpretation of findings. ZDC guided the project and drafted the manuscript. All authors critically reviewed content and approved final version for publication.

\section{References}

Ash BL, Zanatta SD, Williams SJ, Lawrence AJ, Djouma E (2011). The galanin-3 receptor antagonist, SNAP 37889, reduces operant responding for ethanol in alcohol-preferring rats. Regul Pept 166: 59-67.

Ash BL, Quach T, Williams SJ, Lawrence AJ, Djouma E (2014). Galanin-3 receptor antagonism by SNAP 37889 reduces motivation to self-administer alcohol and attenuates cue-induced reinstatement of alcohol-seeking in iP rats. Journal of pharmacological sciences 125: 211-216.

Belfer I, Hipp H, Bollettino A, McKnight C, Evans C, Virkkunen M, et al. (2007). Alcoholism is associated with GALR3 but not two other galanin receptor genes. Genes, brain, and behavior 6: 473-481.

Bilbao A, Serrano A, Cippitelli A, Pavon FJ, Giuffrida A, Suarez J, et al. (2016). Role of the satiety factor oleoylethanolamide in alcoholism. Addiction biology 21: 859-872.

Borroto-Escuela DO, Romero-Fernandez W, Mudo G, Perez-Alea M, Ciruela F, Tarakanov AO, et al. (2012). Fibroblast growth factor receptor 1- 5hydroxytryptamine $1 \mathrm{~A}$ heteroreceptor complexes and their enhancement of hippocampal plasticity. Biol Psychiatry 71: 84-91.

Brabant C, Guarnieri DJ, Quertemont E (2014). Stimulant and motivational effects of alcohol: lessons from rodent and primate models. Pharmacol Biochem Behav 122: 37-52. 
Branchek TA, Smith KE, Gerald C, Walker MW (2000). Galanin receptor subtypes. Trends Pharmacol Sci 21: 109-117.

Breese GR, Baumeister AA, McCown TJ, Emerick SG, Frye GD, Crotty K, et al. (1984). Behavioral differences between neonatal and adult 6-hydroxydopaminetreated rats to dopamine agonists: relevance to neurological symptoms in clinical syndromes with reduced brain dopamine. J Pharmacol Exp Ther 231: 343-354.

Buris L, Csabai G, Fodor M, Varga M (1985). Increase of alcohol dehydrogenase and protein content of liver following chronic ethanol administration. FEBS Lett 183: $143-144$.

Castilla-Ortega E, Pavon FJ, Sanchez-Marin L, Estivill-Torrus G, Pedraza C, Blanco E, et al. (2016). Both genetic deletion and pharmacological blockade of lysophosphatidic acid LPA1 receptor results in increased alcohol consumption. Neuropharmacology 103: 92-103.

Chappell AM, Carter E, McCool BA, Weiner JL (2013). Adolescent rearing conditions influence the relationship between initial anxiety-like behavior and ethanol drinking in male Long Evans rats. Alcohol Clin Exp Res 37 Suppl 1: E394403.

Diaz-Cabiale Z, Parrado C, Vela C, Razani H, Covenas R, Fuxe K, et al. (2005). Role of galanin and galanin(1-15) on central cardiovascular control. Neuropeptides 39: 185-190.

Diaz-Cabiale Z, Parrado C, Narvaez M, Millon C, Puigcerver A, Fuxe K, et al. (2010). Neurochemical modulation of central cardiovascular control: the integrative role of galanin. EXS 102: 113-131.

Diaz-Cabiale Z, Parrado C, Narvaez M, Puigcerver A, Millon C, Santin L, et al. (2011). Galanin receptor/Neuropeptide Y receptor interactions in the dorsal raphe nucleus of the rat. Neuropharmacology $61: 80-86$.

Flores-Burgess A, Millon C, Gago B, Narvaez M, Borroto-Escuela DO, Mengod G, et al. (2017). Galanin (1-15) enhancement of the behavioral effects of Fluoxetine in the forced swimming test gives a new therapeutic strategy against depression. Neuropharmacology 118: 233-241.

Fuxe K, Borroto-Escuela DO, Romero-Fernandez W, Tarakanov AO, Calvo F, Garriga P, et al. (2012). On the existence and function of galanin receptor heteromers in the central nervous system. Front Endocrinol (Lausanne) 3: 127. 
Fuxe K, Marcellino D, Rivera A, Diaz-Cabiale Z, Filip M, Gago B, et al. (2008). Receptor-receptor interactions within receptor mosaics. Impact on neuropsychopharmacology. Brain Res Rev 58: 415-452.

Hedlund PB, Fuxe K (1996). Galanin and 5-HT1A receptor interactions as an integrative mechanism in 5-HT neurotransmission in the brain. Ann N Y Acad Sci 780: 193-212.

Hedlund PB, Yanaihara N, Fuxe K (1992). Evidence for specific N-terminal galanin fragment binding sites in the rat brain. Eur J Pharmacol 224: 203-205.

Jacobowitz DM, Kresse A, Skofitsch G (2004). Galanin in the brain: chemoarchitectonics and brain cartography--a historical review. Peptides 25: 433464 .

Karatayev O, Baylan J, Leibowitz SF (2009). Increased intake of ethanol and dietary fat in galanin overexpressing mice. Alcohol 43: 571-580.

Karatayev O, Baylan J, Weed V, Chang S, Wynick D, Leibowitz SF (2010). Galanin knockout mice show disturbances in ethanol consumption and expression of hypothalamic peptides that stimulate ethanol intake. Alcohol Clin Exp Res 34: 7280.

Koob GF (1992). Drugs of abuse: anatomy, pharmacology and function of reward pathways. Trends Pharmacol Sci 13: 177-184.

Lang R, Gundlach AL, Holmes FE, Hobson SA, Wynick D, Hokfelt T, et al. (2015). Physiology, signaling, and pharmacology of galanin peptides and receptors: three decades of emerging diversity. Pharmacological reviews 67: 118-175.

Leeman RF, Heilig M, Cunningham CL, Stephens DN, Duka T, O'Malley SS (2010). Ethanol consumption: how should we measure it? Achieving consilience between human and animal phenotypes. Addiction biology 15: 109-124.

Leibowitz SF, Avena NM, Chang GQ, Karatayev O, Chau DT, Hoebel BG (2003). Ethanol intake increases galanin mRNA in the hypothalamus and withdrawal decreases it. Physiol Behav 79: 103-111.

Lewis MJ, Johnson DF, Waldman D, Leibowitz SF, Hoebel BG (2004). Galanin microinjection in the third ventricle increases voluntary ethanol intake. Alcohol Clin Exp Res 28: 1822-1828. 
Lewis MJ, Rada P, Johnson DF, Avena NM, Leibowitz SF, Hoebel BG (2005). Galanin and alcohol dependence: neurobehavioral research. Neuropeptides 39: 317321.

Lumeng L, Bosron WF, Li TK (1979). Quantitative correlation of ethanol elimination rates in vivo with liver alcohol dehydrogenase activities in fed, fasted and food-restricted rats. Biochem Pharmacol 28: 1547-1551.

Marcinkiewcz CA, Lowery-Gionta EG, Kash TL (2016). Serotonin's Complex Role in Alcoholism: Implications for Treatment and Future Research. Alcohol Clin Exp Res 40: 1192-1201.

Millon C, Flores-Burgess A, Narvaez M, Borroto-Escuela DO, Santin L, Parrado C, et al. (2015). A role for galanin N-terminal fragment (1-15) in anxiety- and depression-related behaviors in rats. Int $J$ Neuropsychopharmacol 18: 1-13.

Millon C, Flores-Burgess A, Narvaez M, Borroto-Escuela DO, Santin L, Gago B, et al. (2016). Galanin (1-15) enhances the antidepressant effects of the 5-HT1A receptor agonist 8-OH-DPAT: involvement of the raphe-hippocampal 5-HT neuron system. Brain Struct Funct 221(9):4491-4504.

Millon C, Flores-Burgess A, Narvaez M, Borroto-Escuela DO, Gago B, Santin L, et al. (2017). The neuropeptides Galanin and Galanin(1-15) in depression-like behaviours. Neuropeptides 64:39-45.

Mitsukawa K, Lu X, Bartfai T (2008). Galanin, galanin receptors and drug targets. Cell Mol Life Sci 65: 1796-1805.

Paxinos G (1986). The rat Brain in the stereotaxic coodinates. New York: Academic Press edn.

Picciotto MR (2008). Galanin and addiction. Cell Mol Life Sci 65: 1872-1879.

Picciotto MR, Brabant C, Einstein EB, Kamens HM, Neugebauer NM (2010). Effects of galanin on monoaminergic systems and HPA axis: Potential mechanisms underlying the effects of galanin on addiction- and stress-related behaviors. Brain Res 1314: 206-218.

Pierce RC, Kumaresan V (2006). The mesolimbic dopamine system: the final common pathway for the reinforcing effect of drugs of abuse? Neurosci Biobehav Rev 30: 215-238. 
Rada P, Mark GP, Hoebel BG (1998). Galanin in the hypothalamus raises dopamine and lowers acetylcholine release in the nucleus accumbens: a possible mechanism for hypothalamic initiation of feeding behavior. Brain Res 798: 1-6.

Rada P, Avena NM, Leibowitz SF, Hoebel BG (2004). Ethanol intake is increased by injection of galanin in the paraventricular nucleus and reduced by a galanin antagonist. Alcohol 33: 91-97.

Scheller KJ, Williams SJ, Lawrence AJ, Djouma E (2017). The galanin-3 receptor antagonist, SNAP 37889, suppresses alcohol drinking and morphine selfadministration in mice. Neuropharmacology 118: 1-12.

Schneider ER, Rada P, Darby RD, Leibowitz SF, Hoebel BG (2007). Orexigenic peptides and alcohol intake: differential effects of orexin, galanin, and ghrelin. Alcohol Clin Exp Res 31: 1858-1865.

Shephard MD, Penberthy LA, Berry MN (1987). Adaptation of methods for glutamate dehydrogenase and alcohol dehydrogenase activities to a centrifugal analyser: assessment of their clinical use in anoxic states of the liver. J Clin Pathol 40: $1240-1246$.

Simms JA, Steensland P, Medina B, Abernathy KE, Chandler LJ, Wise R, et al. (2008). Intermittent access to $20 \%$ ethanol induces high ethanol consumption in Long-Evans and Wistar rats. Alcohol Clin Exp Res 32: 1816-1823.

Smith KE, Walker MW, Artymyshyn R, Bard J, Borowsky B, Tamm JA, et al. (1998). Cloned human and rat galanin GALR3 receptors. Pharmacology and activation of G-protein inwardly rectifying K+ channels. J Biol Chem 273: 2332123326.

Tarragon E, Balino P, Aragon CM, Pastor R (2012). Ethanol drinking-in-the-dark facilitates behavioral sensitization to ethanol in C57BL/6J, BALB/cByJ, but not in mu-opioid receptor deficient CXBK mice. Pharmacol Biochem Behav 101: 14-23.

Tatemoto K, Rokaeus A, Jornvall H, McDonald TJ, Mutt V (1983). Galanin - a novel biologically active peptide from porcine intestine. FEBS Lett 164: 124-128.

Tsuda K, Tsuda S, Nishio I, Masuyama Y, Goldstein M (1998). Effects of galanin on dopamine release in the central nervous system of normotensive and spontaneously hypertensive rats. American journal of hypertension 11: 1475-1479. 
Vallof D, Ulenius L, Egecioglu E, Engel JA, Jerlhag E (2016). Central administration of the anorexigenic peptide neuromedin $\mathrm{U}$ decreases alcohol intake and attenuates alcohol-induced reward in rodents. Addiction biology 22(3):640-651.

Waters SM, Krause JE (2000). Distribution of galanin-1, -2 and -3 receptor messenger RNAs in central and peripheral rat tissues. Neuroscience 95: 265-271.

\section{Table}

Table 1. Liver ADH activity of animals of two-bottle choice paradigm

\section{ADH Activity}

\begin{tabular}{ccc}
\hline Treatment & Baseline & Veh/EtOH \\
\hline ADH (Ui/g Protein) & $251.4 \pm 14.8$ & $313.1 \pm 10.6^{* *}$ \\
\hline
\end{tabular}

Effects of voluntary ethanol intake in animals of two-bottle choice test over the liver ADH activity. Cerebrospinal fluid-injected rats were used as the vehicle group and naïve rats were used as baseline group. Data are mean \pm SEM ( $n=6-7$ animals per group). ${ }^{*} p<0.01$ versus baseline group according to student's t-test.

\section{Figure Legends}

Figure 1. Experimental design of voluntary ethanol intake.

Figure 2. Dose-response curve of Galanin (1-15) [GAL15] in the two-bottle choice paradigm with $10 \%$ ethanol $(\mathrm{EtOH})$ concentration in rats. GAL15 (at 1 or $3 \mathrm{nmol} / \mathrm{rat}$ ) ( $\mathrm{n}=7-9$ animals per group) was administrated i.c.v. 2, 14 and 24 hours before the measures. Cerebrospinal fluid-injected rats were used as the vehicle group ( $n=15-18$ animals). Vertical bars represent mean \pm SEM of EtOH intake $(\mathrm{g} / \mathrm{Kg} ; \mathrm{a}, \mathrm{b}, \mathrm{c})$, water intake (g/kg; d, e, f), preference EtOH (\%; g, h, i) and Food Intake (g; j, k, l) during the differents periods. (a) " $\mathrm{p}<0.05$ versus rest of the groups (rest of graphs) ${ }^{*} p<0.05$ versus vehicle group according to oneway ANOVA followed by Fisher's LSD test. 
Figure 3. Effect of administration of Galanin (GAL) and Galanin (1-15) [GAL15] in the two-bottle choice paradigm with $10 \%$ ethanol $(\mathrm{EtOH})$ concentration in rats. GAL ( $3 \mathrm{nmol} / \mathrm{rat}$ ) and GAL15 ( $3 \mathrm{nmol} / \mathrm{rat})$ ( $\mathrm{n}=7-13$ animals per group) were administrated i.c.v. 2, 14 and 24 hours before the measures. Cerebrospinal fluid-injected rats were used as the vehicle group ( $n=10-15$ animals). Vertical bars represent mean \pm SEM of EtOH intake $(\mathrm{g} / \mathrm{Kg} ; \mathrm{a}, \mathrm{b}, \mathrm{c})$, water intake $(\mathrm{g} / \mathrm{kg} ; \mathrm{d}$, e, f), preference EtOH (\%; g, h, i) and food Intake $(\mathrm{g} ; \mathrm{j}, \mathrm{k}, \mathrm{I})$ during the differents periods. (a) $p<0.05$ versus vehicle and ${ }^{*} p<0.01$ versus GAL15 $3 \mathrm{nmol}$ group (rest of graphs) $p<0.05$ versus rest of the groups according to one-way ANOVA followed by Fisher's LSD test.

Figure 4. Effects of co-administration of GALR2 receptor antagonist M871 (3 nmol/rat) and Galanin (1-15) [GAL15] ( $\mathrm{n}=7-9$ animals per group) in the twobottle choice paradigm with $10 \%$ ethanol $(\mathrm{EtOH})$ concentration in rats. Treatments were injected i.c.v. 2 hours before the measures. Cerebrospinal fluid-injected rats were used as the vehicle group ( $n=18-20$ animals). Vertical bars represent mean \pm SEM of (a) EtOH intake $(\mathrm{g} / \mathrm{Kg})$, (b) water intake $(\mathrm{g} / \mathrm{kg})$, (c) preference $\mathrm{EtOH}(\%)$ and (d) food Intake (g) during the differents periods. $p<0.05$ versus rest of the groups according to one-way ANOVA followed by Fisher's LSD test.

Figure 5. Effects of Galanin (1-15) [GAL15] in the striatum mRNA expression of C-Fos (a), Rab5 (b), GALR1 (c) and GALR2 (d) in the two-bottle choice paradigm exposed rats. GAL15 was injected i.c.v. 2 hours before the measures. Cerebrospinal fluid-injected rats were used as the vehicle group. Vertical bars represent mean \pm SEM ( $n=5-6$ animals per group). ${ }^{*} p<0.05 ;{ }^{* * *} p<0.01 ;{ }^{* * *} p<0.001$ versus $\mathrm{Veh} / \mathrm{EtOH}$ group according to student's t-test.

Figure 6. Effects of Galanin 1-15 [GAL15] in ethanol-induced locomotors modification in rats. GAL15 (3nmol; i.c.v.) was injected 20 min before the test and acute injection of ethanol $(1.75 \mathrm{~g} / \mathrm{Kg}$; I.P.) was administrated 5 min before the test. Data represents mean \pm SEM ( $n=6-8$ animals per groups) of total distance travelled (a) and mean speed (b) in open field during the 5 min test period. ${ }^{* * *} p<0.001$ according to two-way ANOVA followed by Fisher's LSD test.

Figure 7. Effects of ethanol administration on GALR1 and GALR2 expression in the striatum. Effects of acute i.p. administration of $\mathrm{EtOH}(4 \mathrm{~g} / \mathrm{Kg})$ on $\mathrm{GALR} 1$ (a) and GALR2 (b) mRNA expression in striatum were measured at 0,2 and 4 hours after injection. Data are mean \pm SEM ( $n=4-8$ animals per group). No significant differences were found by one-way ANOVA. 
Figure 1.

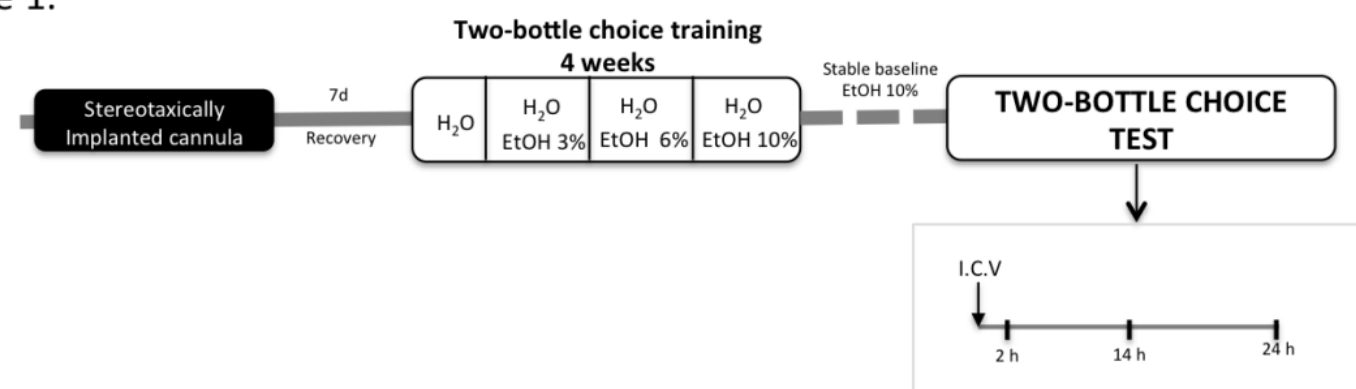

Figure 2. a. EtOH intake $2 \mathrm{hrs}$

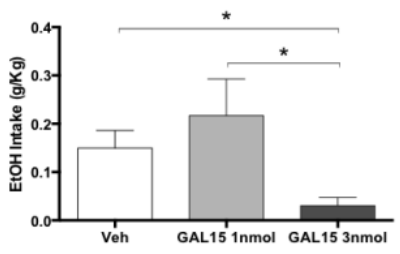

d. Water intake $2 \mathrm{hrs}$

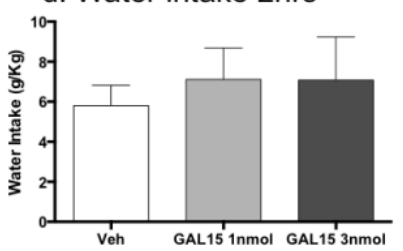

g. EtOH preference $2 \mathrm{hrs}$

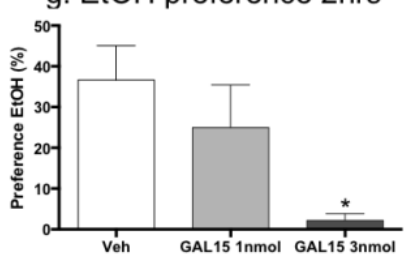

i. Food intake $2 \mathrm{hrs}$

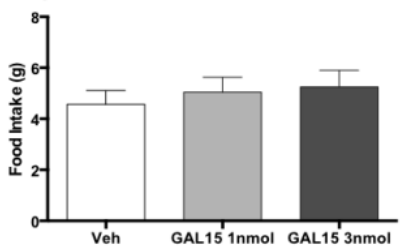

b. EtOH intake $14 \mathrm{hrs}$

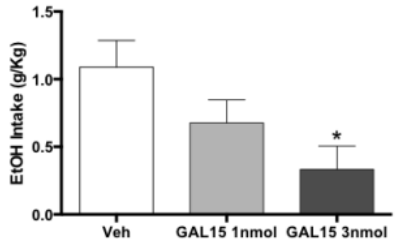

e. Water intake $14 \mathrm{hrs}$

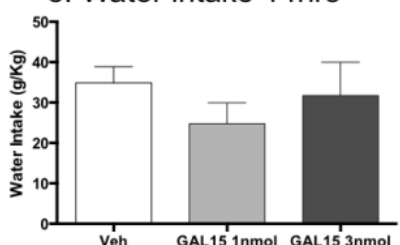

h. EtOH preference $14 \mathrm{hrs}$

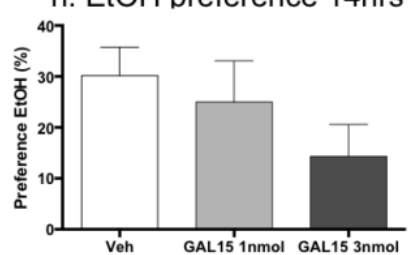

$$
\text { k. Food intake } 14 \mathrm{hrs}
$$

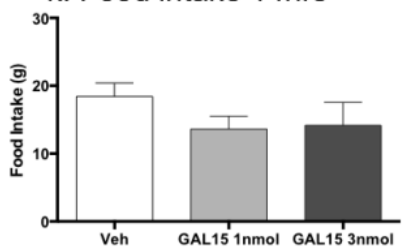

c. EtOH intake 24hrs

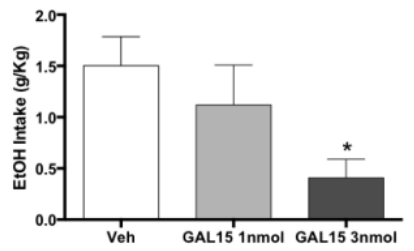

f. Water intake $24 \mathrm{hrs}$

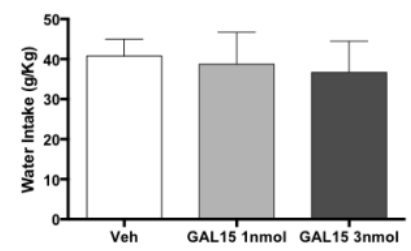

i. EtOH preference 24hrs

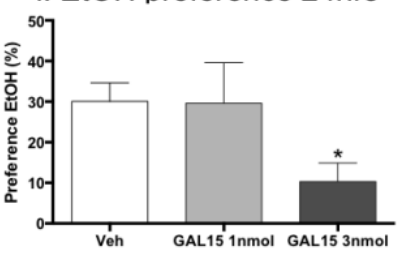

I. Food intake $24 \mathrm{hrs}$

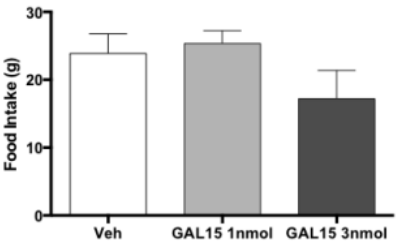


Figure 3.

a. EtOH intake $2 \mathrm{hrs}$

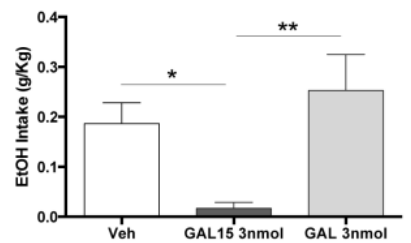

d. Water intake $2 \mathrm{hrs}$

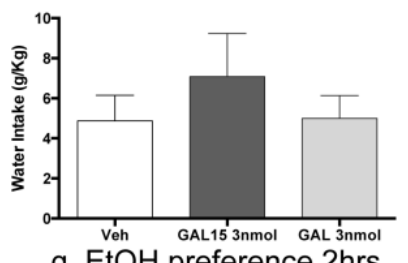

g. EtOH preference $2 \mathrm{hrs}$
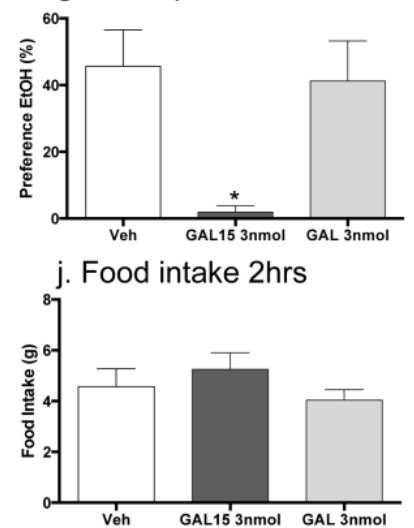

b. EtOH intake $14 \mathrm{hrs}$

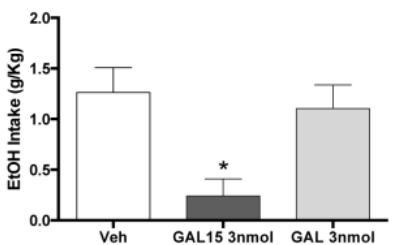

e. Water intake $14 \mathrm{hrs}$
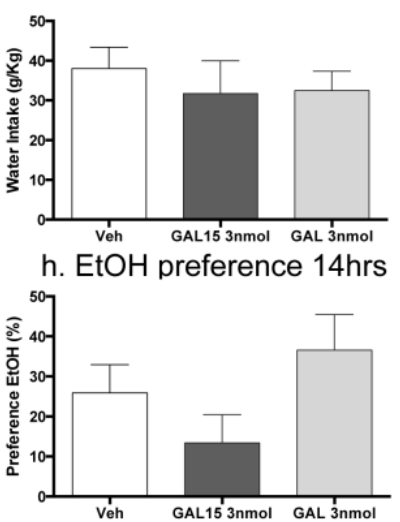

k. Food intake $14 \mathrm{hrs}$

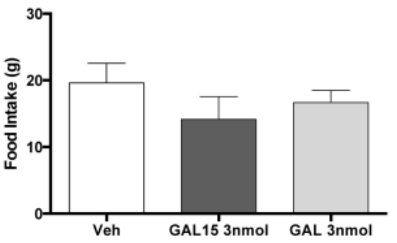

c. EtOH intake 24hrs

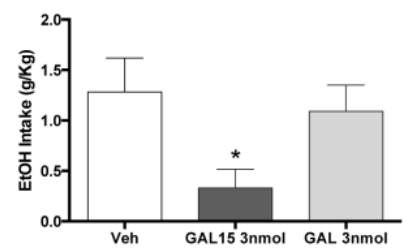

f. Water intake $24 \mathrm{hrs}$

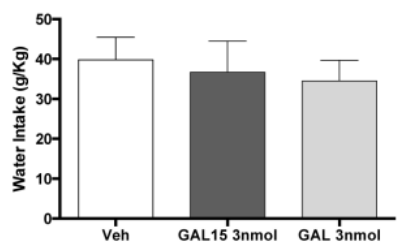

i. EtOH preference $24 \mathrm{hrs}$

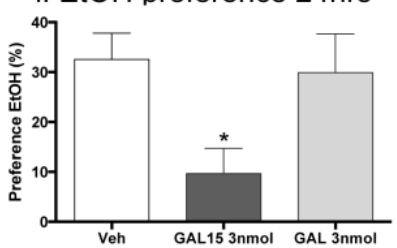

I. Food intake $24 \mathrm{hrs}$

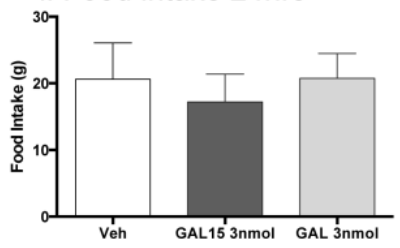

Figure 4.
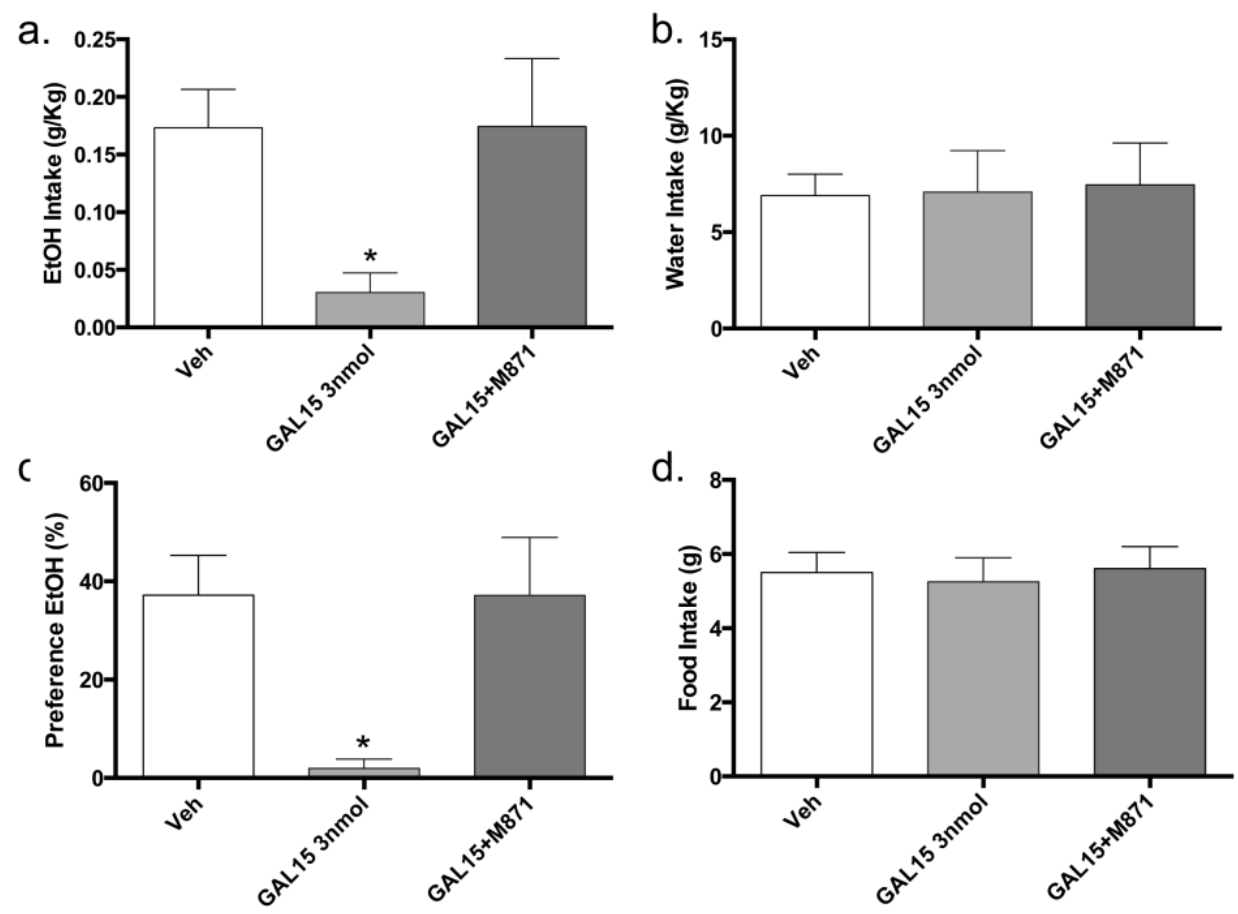
Figure 5.

a.

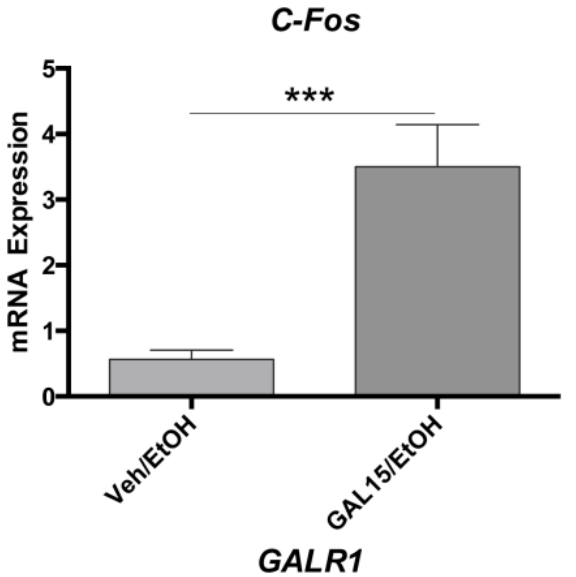

c.

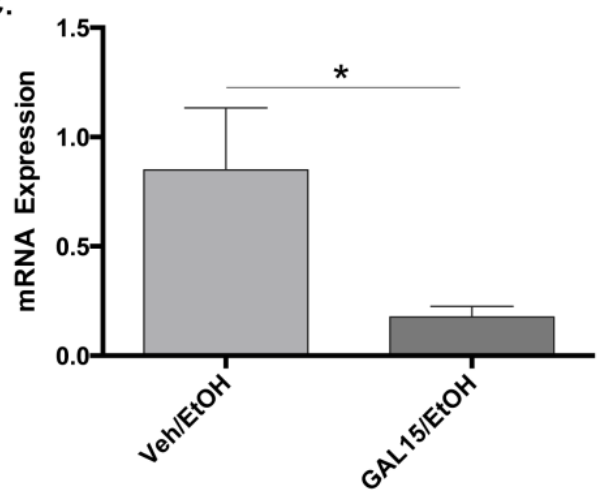

b.

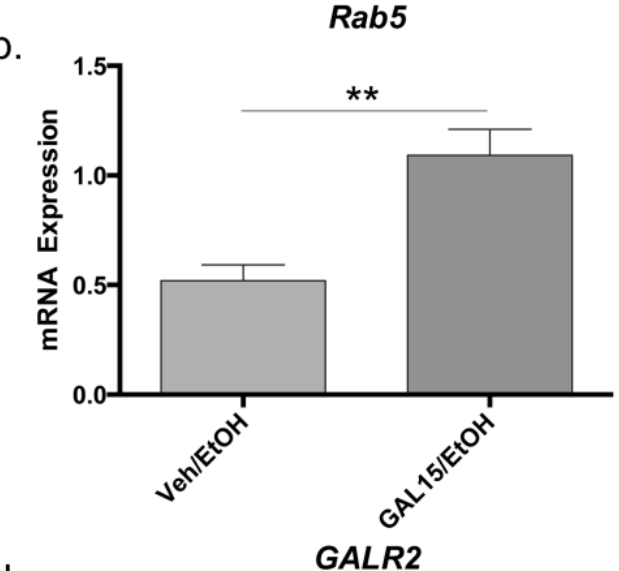

d.

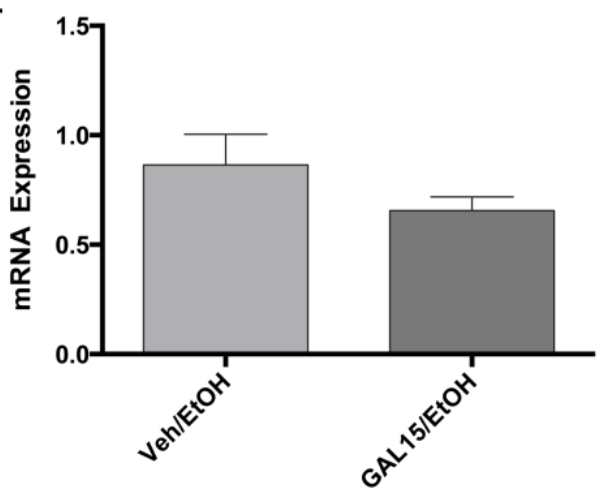

Figure 6.

a.

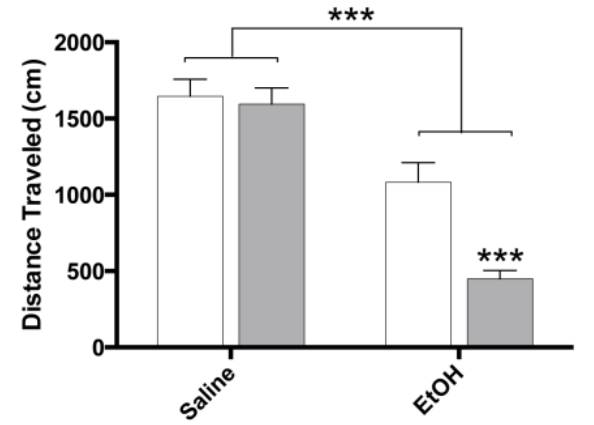

b.

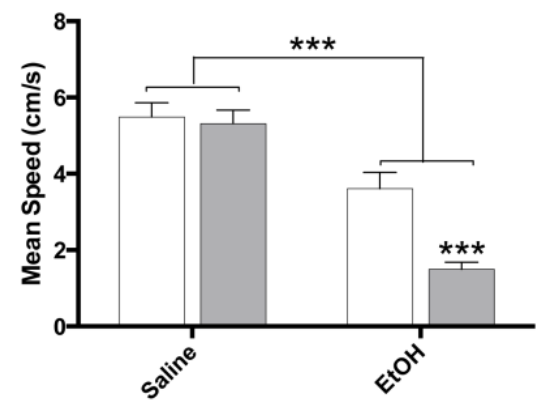

$\square$ Veh

$\square$ GAL15 
Figure 7.
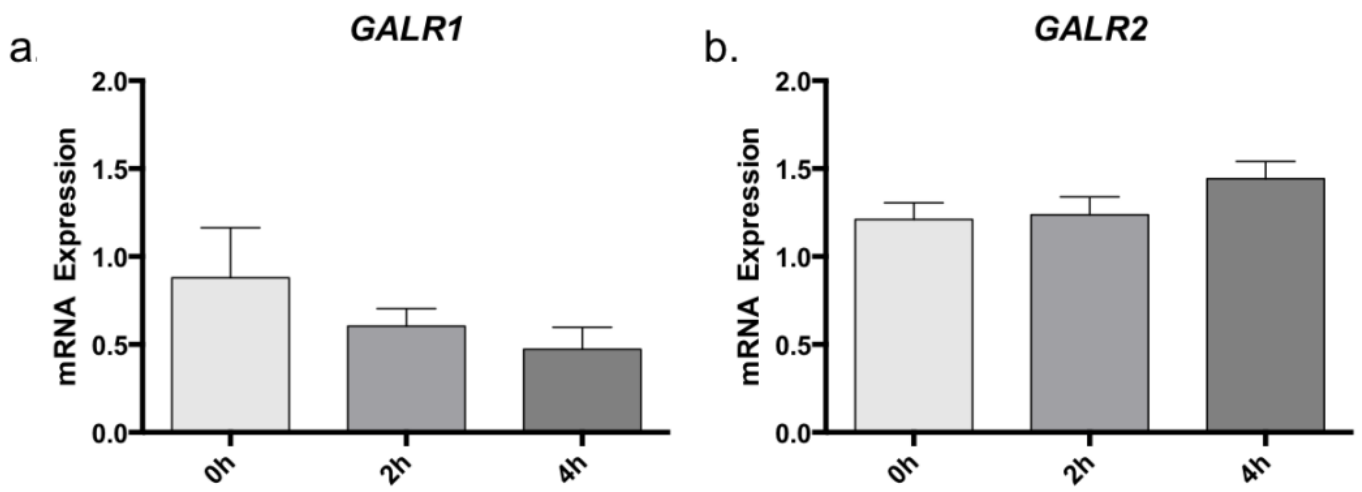